\title{
Correlation of visual area with tremor improvement after MRgFUS thalamotomy in Parkinson's disease
}

\author{
*Yongqin Xiong, MS,' Dongshan Han, BS,1 Jianfeng He, MS, ${ }^{1}$ Rui Zong, MD, ${ }^{2}$ Xiangbing Bian, MS, ${ }^{1}$ \\ Caohui Duan, PhD, ${ }^{1}$ Dekang Zhang, BS, ${ }^{1}$ Xin Zhou, PhD, ${ }^{3}$ Longsheng Pan, MD, ${ }^{2}$ and \\ Xin Lou, MD, PhD'1
}

\begin{abstract}
'Department of Radiology, Chinese PLA General Hospital, Beijing, China; ${ }^{2}$ Department of Neurosurgery, Chinese PLA General Hospital, Beijing, China; and ${ }^{3}$ nnovation Academy for Precision Measurement Science and Technology, The Chinese Academy of Sciences-Wuhan National Laboratory for Optoelectronics, Wuhan, China
\end{abstract}

\begin{abstract}
OBJECTIVE MRI-guided focused ultrasound (MRgFUS) thalamotomy is a novel and minimally invasive alternative for medication-refractory tremor in Parkinson's disease (PD). However, the impact of MRgFUS thalamotomy on spontaneous neuronal activity in PD remains unclear. The purpose of the current study was to evaluate the effects of MRgFUS thalamotomy on local fluctuations in neuronal activity as measured by the fractional amplitude of low-frequency fluctuations (fALFF) in patients with PD.

METHODS Participants with PD undergoing MRgFUS thalamotomy were recruited. Tremor scores were assessed before and 3 and 12 months after treatment using the Clinical Rating Scale for Tremor. MRI data were collected before and 1 day, 1 week, 1 month, 3 months, and 12 months after thalamotomy. The fALFF was calculated. A whole-brain voxelwise paired t-test was used to identify significant changes in fALFF at 12 months after treatment compared to baseline. Then fALFF in the regions with significant differences were extracted from fALFF maps of patients for further one-way repeated-measures ANOVA to investigate its dynamic alterations. The association between fALFF changes induced by thalamotomy and tremor improvement were evaluated using the nonparametric Spearman rank test.
\end{abstract}

RESULTS Nine participants with PD (mean age \pm SD $64.7 \pm 6.1$ years, 8 males) were evaluated. Voxel-based analysis showed that fALFF in the left occipital cortex (Brodmann area 17 [BA17]) significantly decreased at 12 months after thalamotomy compared to baseline (voxel $p<0.001$, cluster $p<0.05$ family-wise error [FWE] corrected). At baseline, fALFF in the left occipital BA17 in patients was elevated compared with that in 9 age- and gender-matched healthy subjects $(p<$ $0.05)$. Longitudinal analysis displayed the dynamic changes of fALFF in this region $\left(F_{(5,40)}=3.61, p=0.009\right)$. There was a significant positive correlation between the falling trend in fALFF in the left occipital BA17 and hand tremor improvement after treatment over 3 time points (Spearman's rho $=0.44, p=0.02$ ).

CONCLUSIONS The present study investigated the impact of MRgFUS ventral intermediate nucleus thalamotomy on spontaneous neural activity in medication-refractory tremor-dominant PD. The visual area is, for the first time, reported as relevant to tremor improvement in PD after MRgFUS thalamotomy, suggesting a distant effect of MRgFUS thalamotomy and the involvement of specific visuomotor networks in tremor control in PD.

https://thejns.org/doi/abs/10.3171/2021.3.JNS204329

KEYWORDS Parkinson's disease; MRgFUS; functional neurosurgery; fALFF

$\mathrm{P}$ ARKINSON's disease (PD) is the second most common neurodegenerative disorder, characterized by degeneration of dopaminergic neurons in the substantia nigra., ${ }^{1,2}$ Tremor is one of the cardinal symptoms of $\mathrm{PD}$, presenting in $75 \%$ to $100 \%$ of patients during the course of their disease. ${ }^{3,4}$ Tremor can lead to a range of degrees of disability at home and in the workplace, affecting employment and potentially causing social isolation. ${ }^{5}$ However, although the bradykinesia and rigidity of PD reliably respond to dopamine therapy, the response of par-

ABBREVIATIONS 3D FSPGR = three-dimensional fast spoiled gradient recalled; ALFF = amplitude of low-frequency fluctuations; BA17 = Brodmann area $17 ; \mathrm{BOLD}=$ blood oxygen level-dependent; CRST = Clinical Rating Scale for Tremor; EPI = echo planar imaging; FA = flip angle; fALFF = fractional ALFF; FWE = family-wise error; MNI = Montreal Neurological Institute; MRgFUS = magnetic resonance imaging-guided focused ultrasound; PD = Parkinson's disease; rs-fMRI = resting-state functional MRI; UPDRS = Unified Parkinson's Disease Rating Scale; VIM = ventral intermediate.

SUBMITTED December 15, 2020. ACCEPTED March 1, 2021.

INCLUDE WHEN CITING Published online September 3, 2021; DOI: 10.3171/2021.3.JNS204329.

${ }^{*}$ Y.X. and D.H. contributed equally to this work. 
kinsonian tremor to dopamine treatment is inconsistent and unpredictable..$^{6,7}$

Magnetic resonance imaging-guided focused ultrasound (MRgFUS) thalamotomy, a novel and minimally invasive technology for reducing medication-refractory tremor in PD, enables focal ventral intermediate (VIM) nucleus ablation by generating high-intensity ultrasound energy without cranium opening, anesthesia, or ionizing radiation. ${ }^{8-10}$ This technology has led to a renaissance of intracranial lesional functional neurosurgeries. However, little is known about the impact of a focal, deliberately ablated VIM lesion on brain function, and the mechanism underlying thalamotomy-induced tremor arrest remains unclear. A previous study based on a diffusion tensor MRI technique investigated the effects of MRgFUS thalamotomy on white matter integrity in PD and found longterm damage in the ablated core and the tract connecting the thalamus to the red nucleus. ${ }^{11}$ However, the effects of MRgFUS thalamotomy on spontaneous neuronal activity in PD have never been demonstrated.

The amplitude of low-frequency fluctuations (ALFF) and fractional ALFF (fALFF), measures of the magnitude of spontaneous blood oxygen level-dependent (BOLD) signal oscillations, have been proposed as suggestive of spontaneous neuronal activity. ${ }^{12-14}$ As a normalized index of ALFF, fALFF can provide more sensitive and specific measures of spontaneous neuronal activity by suppressing nonspecific signal components. ${ }^{15}$ The regional differences in fALFF have been reported to be reliable for discriminating PD patients from healthy controls. ${ }^{16-18}$ These findings indicate the potential of fALFF as a PD biomarker to assess treatment response and to clarify the basis of tremor suppression conveyed by MRgFUS thalamotomy.

In the present study, we aimed to assess the effects of MRgFUS thalamotomy on local BOLD oscillations as measured by fALFF in PD patients. First, we explored the changes in fALFF in PD patients at 12 months after MRgFUS thalamotomy compared to baseline based on whole-brain voxel-wise analysis, which is a data-driven approach allowing one to detect regional changes throughout the brain without any a priori hypothesis regarding loci. Then we investigated the longitudinal dynamic alterations of fALFF in the regions with a significant difference. We also investigated fALFF changes within the lesion center based on region-of-interest analysis. We hypothesized that MRgFUS thalamotomy could exert modulatory effects on spontaneous neuronal activity in remote areas beyond the target.

\section{Methods \\ Participants}

Ten right-handed patients with medication-refractory tremor-dominant PD underwent unilateral MRgFUS thalamotomy targeting the VIM nucleus contralateral to the most severely affected extremity. All patients had been diagnosed with PD by an experienced movement disorder neurologist (R.Z.). Dominance of the tremor was defined by a ratio of mean Unified Parkinson's Disease Rating Scale (UPDRS) tremor scores to the mean UPDRS postural instability/gait disorder scores $\geq 1.5 .^{19}$ Details of the procedure were consistent with previous reports. ${ }^{20-23}$ Clinical evaluations were performed at baseline and 3 and 12 months after thalamotomy. MRI data were collected before and 1 day, 1 week, 1 month, 3 months, and 12 months after thalamotomy. One patient did not complete the series of posttreatment clinical evaluations and MRI scans after treatment; thus, 9 patients were included in the analysis. Nine age- and gender-matched right-handed healthy subjects were recruited as controls (mean age \pm SD $60.1 \pm$ 6.7 years, range $51-70$ years, 8 males). The study was approved by the ethics committees of the Chinese PLA General Hospital. All patients gave informed consent before the study.

\section{Clinical Evaluation}

Tremor assessments were performed in the off-medication state (i.e., overnight withdrawal of antiparkinsonian drugs). ${ }^{24}$ The tremor score for the hand contralateral (treated) to the thalamotomy was calculated using a subset of scores derived from the Clinical Rating Scale for Tremor (CRST) Part A, which evaluates rest, posture, and action components of hand tremor, and CRST Part $\mathrm{B}$, which assesses five tasks involving handwriting (dominant hand only), drawing, and pouring. The treated hand tremor subscore is on a scale ranging from 0 to 32 when the dominant hand is treated and a scale ranging from 0 to 28 when the nondominant hand is treated. A higher score indicates more severe tremor. The rest, postural, and action tremor components of the treated hand (scale of $0-4$ for each component) were separately assessed.

\section{MRI Acquisition}

MRI data were collected on a Discovery MR750 scanner (General Electric). Patients were instructed to relax, keep their eyes closed, and to not sleep or think specifically during MRI acquisition. Ear plugs and tight but comfortable foam padding were used to reduce noise and head motion.

The resting-state functional MRI (rs-fMRI) data were acquired using echo planar imaging (EPI) sequences with the following parameters: TR $2000 \mathrm{msec}$, TE $30 \mathrm{msec}$, flip angle (FA) $90^{\circ}$, FOV $240 \times 240 \mathrm{~mm}$, matrix $64 \times 64$, slice thickness $3.5 \mathrm{~mm}$, slice gap $0.5 \mathrm{~mm}$, and 36 interleaved slices. Each functional run produced 180 volumes.

The high-resolution structural data were obtained using a three-dimensional fast spoiled gradient recalled (3D FSPGR) sequence with the following parameters: TR $6.656 \mathrm{msec}$, TE $2.928 \mathrm{msec}$, TI $800 \mathrm{msec}$, FA $7^{\circ}$, FOV $256 \times 256 \mathrm{~mm}$, matrix $256 \times 256$, slice thickness $1 \mathrm{~mm}$, and 192 contiguous sagittal slices.

\section{Imaging Analysis \\ Imaging Preprocessing of rs-fMRI}

MRI scans from patients with right thalamotomy $(n=$ 3) were flipped along the x-axis so that all ablated lesions appeared to be on the left hemisphere. Image preprocessing was conducted using an rs-fMRI data analysis toolkit (REST version 1.2, http://www.restfmri.net). ${ }^{25}$ The procedures consist of the following steps: 1) discarding the first 10 time points of functional images considering the 
effects of scanning instability and patient adaptation; 2) slice timing across slices; 3 ) head motion correction (no scans were discarded according to the criteria of head displacement $>2.5 \mathrm{~mm}$ or angular rotation $>2.5^{\circ}$ in any direction); 4) coregistration and spatial normalization to the standard Montreal Neurological Institute (MNI) EPI template; 5) spatial smoothing with a 6-mm full width at half maximum Gaussian kernel; 6) removing the linear trend; and 7) regressing the six rigid parameters of head motion, white matter signal, and cerebrospinal fluid signal.

\section{fALFF Calculation}

After preprocessing, a temporary bandpass range of 0.01-0.08 Hz was set to filter the BOLD time series for each voxel to remove the very-low frequency and high frequency noise. ${ }^{26}$ Then the filtered time series were transformed to the frequency domain using a fast Fourier transformation to obtain the power spectrum. Next, the ALFF for each voxel was calculated as the averaged square-rooted power spectrum within the frequency band of $0.01-0.08 \mathrm{~Hz}$. Finally, a division of the ALFF within the specified frequency band by the entire frequency range was computed at each voxel to yield the fALFF, which could provide a more stable and accurate measure of spontaneous neural activity. ${ }^{15}$ The fALFF maps were standardized into z-score maps to minimize the global effects of variability.

\section{Voxel-Based Analysis}

We first performed a whole-brain voxel-wise paired ttest using the SPM package (http://www.fil.ion.ucl.ac.uk/ $\mathrm{spm} /$ ) to identify significant changes in the fALFF at 12 months after treatment compared to baseline (within the gray matter mask). Brain regions were reported at a threshold of voxel-wise $\mathrm{p}<0.001$ and cluster level $\mathrm{p}<0.05$ with family-wise error (FWE) correction. The fALFF values in the region with a significant difference were extracted from the fALFF maps of patients acquired at each time point and the fALFF maps of healthy controls. One-way repeated-measures ANOVA with Mauchly's sphericity test and post hoc paired Student t-test using Bonferroni corrections for five comparisons $(\alpha=0.01)$ were conducted to determine the dynamic alterations of the extracted fALFF over six time points in patients using SPSS (version 19.0, IBM Corp.). The extracted baseline fALFF and 12-month postoperative fALFF of patients were separately compared to the extracted fALFF values of healthy controls to determine if MRgFUS thalamotomy normalized the fALFF in the identified region in patients. The association between the longitudinal changes of fALFF in the regions with a significant difference induced by thalamotomy and longitudinal changes of the treated hand tremor scores over three time points (i.e., before and 3 and 12 months after treatment) was evaluated using the nonparametric Spearman rank test. A statistical significance threshold was set at $\mathrm{p}<0.05$.

\section{Lesion Analysis}

Ablated lesion centers defined by Wintermark et al. ${ }^{27}$ were manually delineated by a neuroradiologist (J.H.) on
TABLE 1. Demographic characteristics and clinical data for PD patients

\begin{tabular}{lc}
\hline \multicolumn{1}{c}{ Variable } & Value \\
\hline No. of patients (M/F) & $9(8 / 1)$ \\
\hline Mean age in yrs, \pm SD (range) & $64.7 \pm 6.1(53-75)$ \\
\hline Median duration of disease in yrs (range) & $6(3-27)$ \\
\hline Thalamotomy side & 6 \\
\hline Lt & 3 \\
\hline Rt & \\
\hline $\begin{array}{l}\text { Median treated hand tremor score off } \\
\text { medication (range) }\end{array}$ & $17(12-25)$ \\
\hline Baseline & $4(0-20)$ \\
\hline 3 mos & $3(1-8)$ \\
\hline 12 mos
\end{tabular}

the 1-day postprocedure 3D FSPGR images using ITKSnap (www.itksnap.org). The delineated lesions and corresponding 3D FSPGR images were coregistered to the standard MNI T1 template by using SPM12 toolbox (http:// www.fil.ion.ucl.ac.uk/spm/). The quality of registration was visually confirmed. Then the right-sided lesions $(\mathrm{n}=$ 3 ) were flipped along the x-axis. All thalamotomy lesions were summed to generate a lesion mask. The average fALFF values within the lesion were extracted from individual fALFF maps obtained at each session. One-way repeated-measures ANOVA was conducted to assess the dynamic alterations of AALFF within the lesion, and the Bonferroni method corrected for multiple comparisons. The correlation between changes of fALFF within the lesion and changes in the treated upper limb tremor scores was assessed using the nonparametric Spearman rank test.

\section{Results \\ Participant Demographics}

Nine patients (mean age $64.7 \pm 6.1$ years, range 53-75 years, 8 males) with medication-refractory tremor-dominant PD undergoing MRgFUS VIM thalamotomy were included in the analysis. The demographic and clinical data are summarized in Table 1. Treated hand tremor significantly improved at 3 and 12 months after MRgFUS thalamotomy (Friedman test $\chi^{2}[2]=11.03, p=0.004$; post hoc Wilcoxon signed-rank tests using Bonferroni correction for two comparisons, $\alpha=0.025$; Fig. 1).

\section{Impact of MRgFUS Thalamotomy on Low-Frequency Fluctuations}

Voxel-wise paired t-test revealed a significant decrease of fALFF in the left occipital Brodmann area 17 (BA17; voxel $\mathrm{p}<0.001$, cluster $\mathrm{p}<0.05 \mathrm{FWE}$ corrected; peak MNI coordinates: $\mathrm{x}=-12, \mathrm{y}=-90, \mathrm{z}=0$; cluster size $=29$ voxels; peak $\mathrm{T}=7.83$; Fig. $2 \mathrm{~A}$ ) at 12 months after the procedure compared to baseline. Longitudinal analysis showed the decreasing shift toward normality during follow-up after MRgFUS thalamotomy $(\mathrm{F}=3.61, \mathrm{p}=0.009$; post hoc paired Student t-test using Bonferroni correction for five 

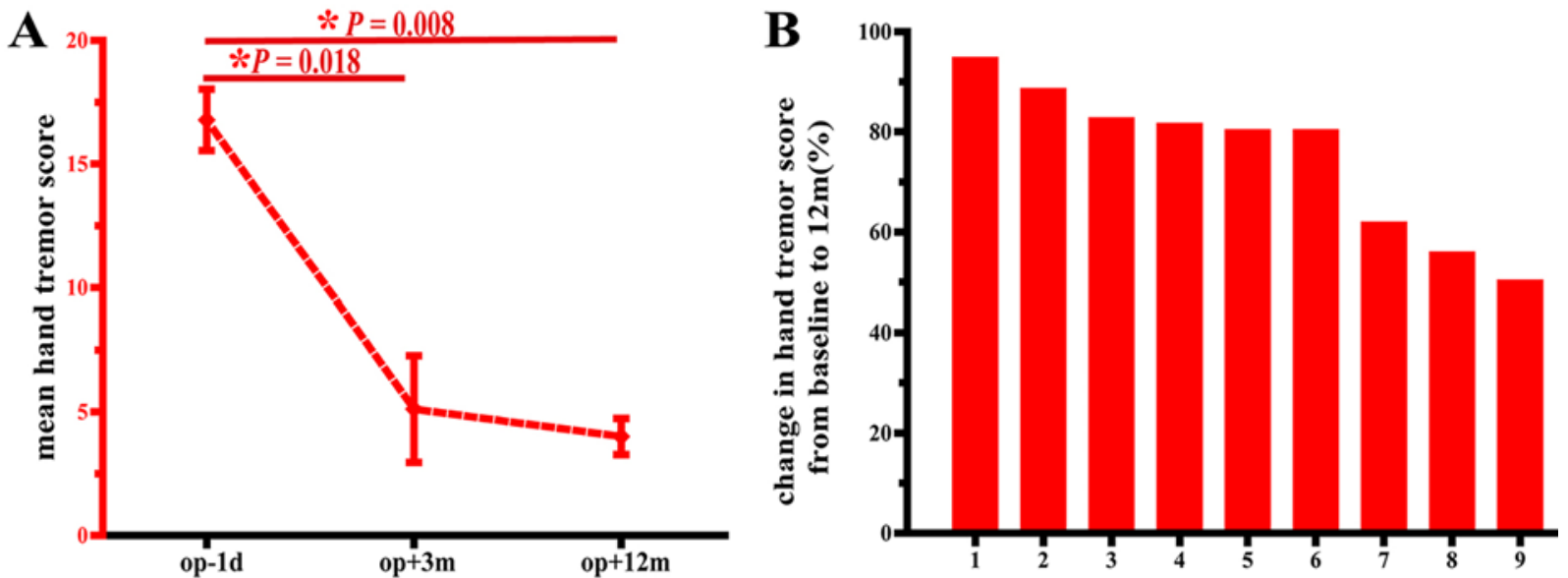

FIG. 1. Tremor scores for the treated hand evaluated according to the CRST. A: Tremor score for the treated hand before and 3 and 12 months after thalamotomy. *Bonferroni correction $(\alpha=0.025)$. B: Tremor improvement ratio at 12 months after thalamotomy for all 9 participants (represented on $x$-axis). $-1 d=$ minus 1 day; $+3 m=$ plus 3 months; $+12 \mathrm{~m}=$ plus 12 months; op = operation. Figure is available in color online only.

comparisons, $\alpha=0.01$; Fig. 2B). At baseline, fALFF in the left occipital BA17 in patients was elevated compared with that in age- and gender-matched healthy subjects, but 12 months after thalamotomy fALFF in the left occipital BA17 did not show a significant difference compared with healthy subjects (Fig. 3). A significant correlation was identified between the decreasing trend of fALFF in the left occipital BA17 and hand tremor improvement after MRgFUS thalamotomy for three time points (Spearman's rho $=0.44, p=0.02 ;$ Fig. 4A). Further analysis of different types of tremor also showed significant positive correlations between the falling trend of fALFF in the left BA17 and rest, postural, or action tremor improvement (rest tremor: Spearman's rho $=0.47, \mathrm{p}=0.01$; postural tremor: Spearman's rho $=0.59, \mathrm{p}=0.001$; action tremor: Spearman's rho $=0.46, \mathrm{p}=0.01$; Fig. 4B-D).

\section{Lesion Analysis}

The volume of lesion centers at 1 day after thalamotomy ranged from 60 to $140 \mathrm{~mm}^{3}$ (mean $94.4 \pm 26 \mathrm{~mm}^{3}$ ). The fALFF within the lesion did not show a significant change after the procedure. The fALFF within the lesion did not correlate with tremor improvement after thalamotomy.
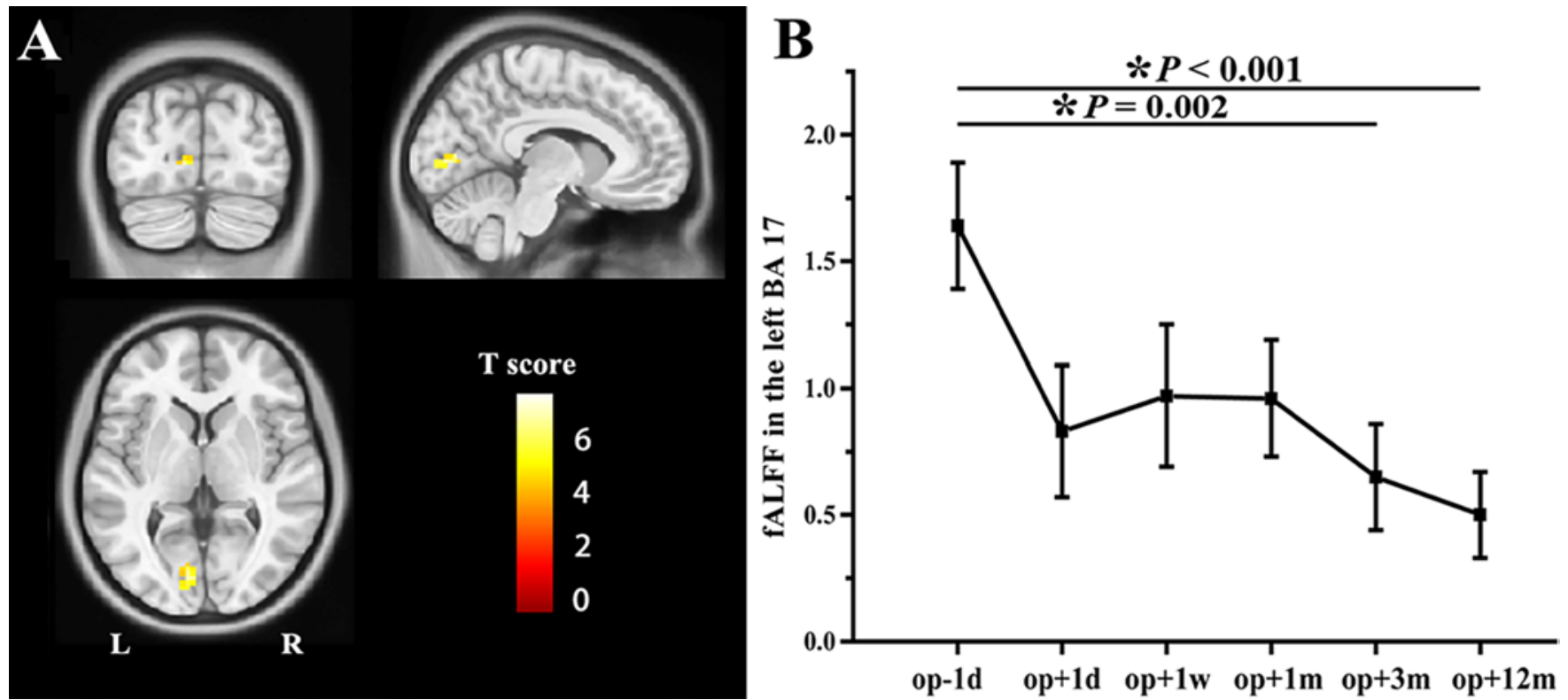

FIG. 2. Impact of MRgFUS thalamotomy on low-frequency fluctuations. A: The colored region represents a significant decrease of fALFF in the left occipital BA17 at 12 months after treatment compared to preoperation (voxel $p<0.001$, cluster $p<0.05$ FWE corrected). Color bar shows T score. B: The dynamic alterations of fALFF in the left occipital BA17. *Bonferroni correction $(\alpha=0.01)$. $+1 \mathrm{w}=$ plus 1 week. Figure is available in color online only. 


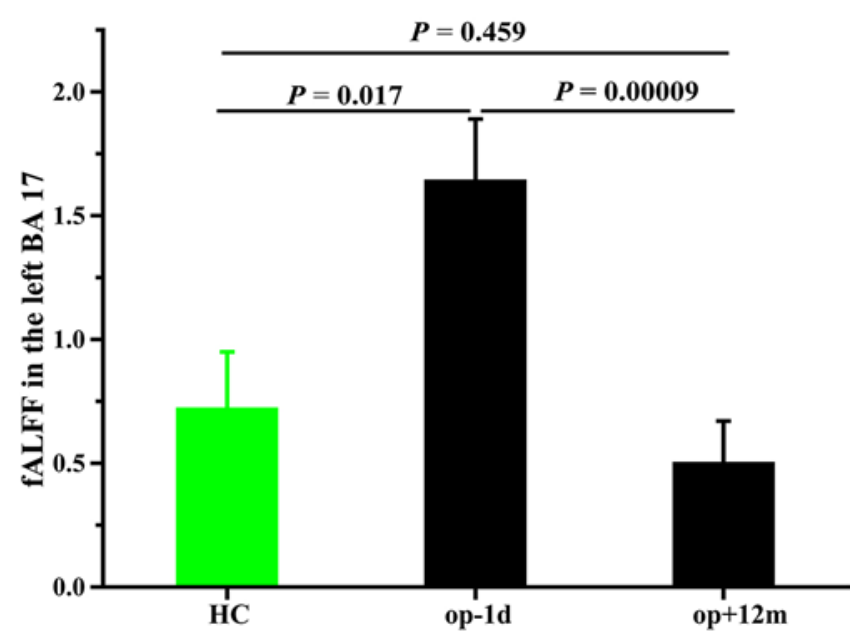

FIG. 3. Difference in fALFF in the left occipital BA17 between healthy controls $(\mathrm{HC})$ and patients before and 12 months after thalamotomy. Figure is available in color online only.

\section{Discussion}

To the best of our knowledge, this is the first investigation to assess the effects of MRgFUS thalamotomy on spontaneous neuronal activity in medication-refractory tremor-dominant PD. We found that MRgFUS thalamotomy results in a significant decrease of fALFF in the left occipital cortex (BA17) in PD, which correlates with tremor improvement.

ALFF or fALFF abnormality has been detected in PD. Altered ALFF in the precentral gyrus, supplementary motor cortex, midbrain, and cerebellum and altered fALFF in the cerebellum, left inferior temporal gyrus, right inferior parietal lobule, and right middle frontal gyrus have been reported in PD. ${ }^{17,28,29}$ In addition, different patterns of spontaneous neuronal activity have been reported in postural instability/gait difficulty and tremor-dominant subtypes of PD..$^{30}$ A recent longitudinal study further found that fALFF in the right middle temporal gyrus and right middle occipital gyrus increased while fALFF in the right cerebellum, right thalamus, right striatum, left superior
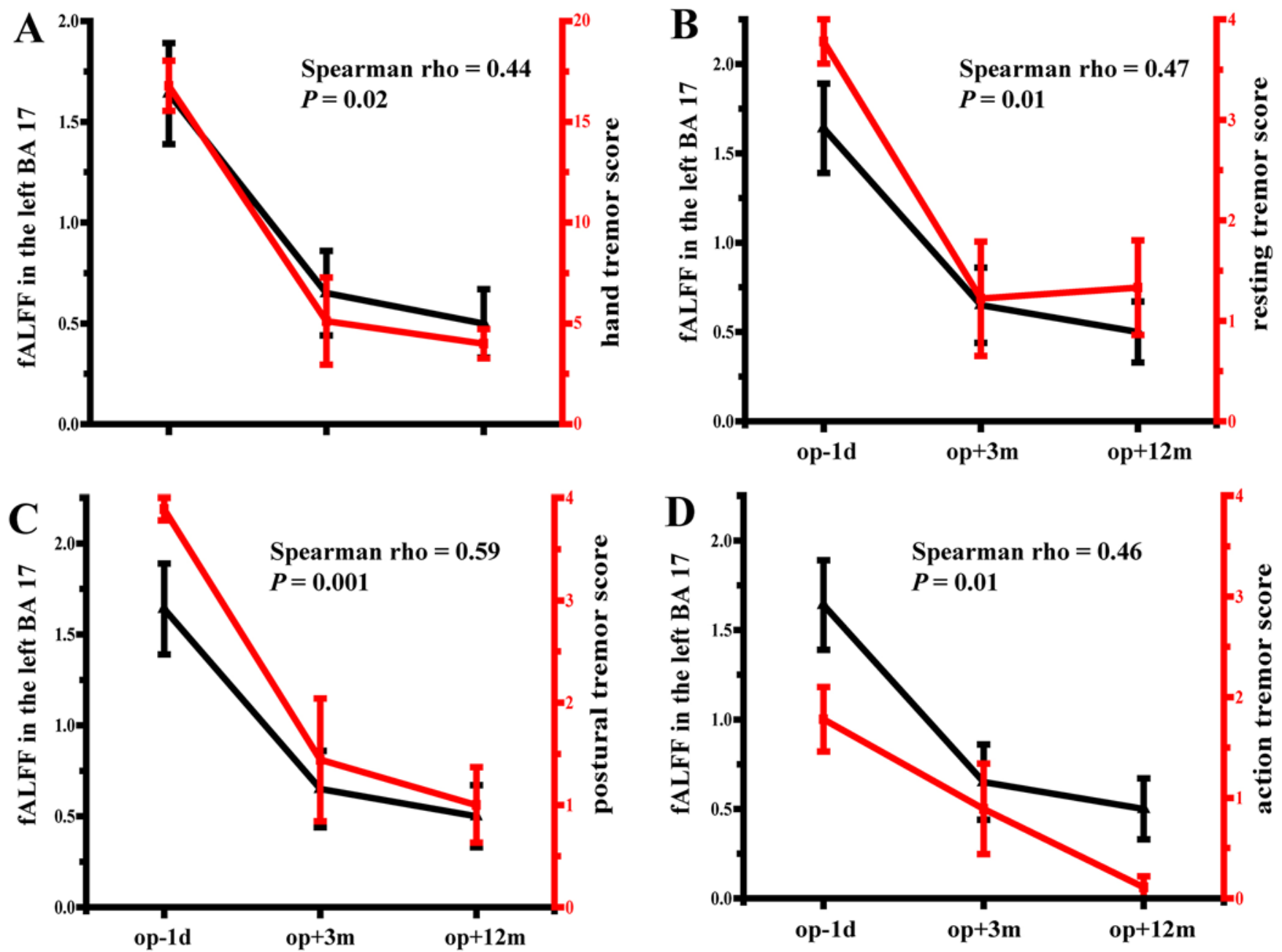

FIG. 4. A significant positive correlation between the falling trend of fALFF in the left occipital BA17 and tremor improvement.

A: Correlation with the hand tremor score. B: Correlation with resting tremor score. C: Correlation with the postural tremor score.

D: Correlation with the action tremor score. Figure is available in color online only. 
parietal lobule, left inferior parietal lobule, left precentral gyrus, and left postcentral gyrus decreased as the disease progressed..$^{18}$ These findings suggest that the pathophysiology of PD involves multiple brain regions besides the ones within the motor network and that ALFF or fALFF could be used as a biomarker for evaluating and monitoring PD.

The current study aimed to investigate the impacts of MRgFUS thalamotomy on local fluctuations in neuronal activity as measured by fALFF in patients with medication-refractory tremor-dominant PD and to learn if the changes of low-frequency fluctuations could underlie the neural substrate of tremor control induced by thalamotomy. Our results showed that MRgFUS thalamotomy leads to a restoration of fALFF in the visual area and that the restoration is correlated with tremor improvement induced by the procedure. This is a novel and interesting finding. Involvement of the left occipital BA17 suggests a remote neuromodulatory effect of MRgFUS thalamotomy. Concerning the distant effects of MRgFUS thalamotomy, previous studies using structural diffusion tensor imaging data or rs-fMRI data combined with the graph theory method have shown the white matter integrity and pathlength changes in distant areas after thalamotomy in patients with essential tremor. ${ }^{31,32}$

Abnormal activity in the visual area has been reported in PD using different methods. For instance, Zhang et al. reported an increased weighted degree centrality in occipital (calcarine and cuneus) regions in patients with PD and revealed that regional efficiency of the brain network involved in the calcarine sulcus as well as the basal ganglia and cerebellum were capable of distinguishing PD subtypes. ${ }^{33,34}$ Göttlich et al. found that PD patients had lower connectedness in the calcarine cortex and that the interaction of the visual network with other brain networks decreased. ${ }^{35} \mathrm{In}$ a recent hybrid ${ }^{18} \mathrm{~F}-\mathrm{FDG}$-PET/MRI study, an increase of metabolic activity in the calcarine cortex was observed after MRgFUS subthalamotomy in PD patients. ${ }^{36}$ However, abnormality of the visual area or visual network has often been linked to the visual problems of PD patients, such as visual hallucinations. The relationship between the visual network and tremor was barely mentioned in the literature except in one study that found that functional connectivity between visual areas and the putamen was correlated with tremor scores in tremor-dominant PD. ${ }^{37}$ According to our findings, the role of visual areas or the visual network in tremor generation and further tremor control after thalamotomy may be underestimated.

It has been postulated that there must be a series of cortical-cortical connections from visual areas to motor ones for the sensory guidance of movement of the hands and fingers. ${ }^{38}$ In line with our findings, previous studies in essential tremor have found a link between visual areas or the visual network and the tremor arrest induced by VIM radiosurgery. ${ }^{39}$ Tuleasca et al. reported a correlation between a decrease in gray matter density in the occipital cortex (BA19) at 1 year after VIM radiosurgery and tremor improvement ${ }^{40}$ and found that gray matter density in the right BA18 at baseline can predict tremor control at 1 year after VIM radiosurgery. ${ }^{41}$ By using group-level independent component analysis, they further determined that interconnectivity between visual and motor areas was correlated with tremor control after VIM radiosurgery. ${ }^{42}$ More recently, these authors used a coactivation pattern analysis to investigate the dynamic interactions of the right extrastriate cortex and found that tremor improvement in patients with essential tremor after stereotactic radiosurgery thalamotomy was correlated with changes in the dynamic functional connectivity of the extrastriate visual system. ${ }^{43}$ These findings support the role of visual areas or a visual network in tremor generation and further suppression after treatment.

However, how a focal deliberate VIM nucleus lesion induces structural and functional changes in visual areas and its relevance to tremor control remain unclear. One possibility could be the calibration of a cerebello-visualmotor network. It has been postulated that visual areas connect to motor ones through pontine cells and the cerebellar cortex. ${ }^{38} \mathrm{~A}$ recent study in essential tremor has reported that tremor improvement after VIM radiosurgery correlated with interconnectivity strength between motor areas and the cerebellum lobule with the visual area, suggesting the presence of a cerebello-visuo-motor functional network related to clinical benefits. ${ }^{42}$ Another possibility would be the regulation of the visual corticostriatal system. It has been hypothesized that the striatum receives inputs from the visual cortex then projects to the pallidonigral complex to mediate visual-motor associations and visually guided reaching. ${ }^{44}$ The identified correlation between putamen-visual area functional connectivity and tremor scores in tremor-dominant PD suggests the involvement of the visual corticostriatal system in Parkinson tremor. ${ }^{37}$ Further studies are needed to address these issues. Furthermore, Tuleasca et al. have raised the question of whether visual areas should be targeted in essential tremor. And, the same question remains open in PD.

Interestingly, different visual areas are reported to link with tremor arrest after VIM thalamotomy in essential tremor and PD. The visual association areas (BA18 and BA19) are reported to be involved in tremor arrest after VIM radiosurgery in essential tremor. ${ }^{40,41}$ However, the primary visual area (BA17) is, for the first time, identified as relevant to tremor improvement after MRgFUS thalamotomy in tremor-dominant PD. Is there a possibility that tremor control in essential tremor and PD after VIM thalamotomy may recruit different visual networks? Does it have a role in discriminating essential tremor and tremor-dominant PD? Caution should be exercised because different methods may contribute to these differences. More research is needed in the future.

We did not find significant fALFF changes within the lesion center, which was expected because rs-fMRI is insensitive to the small subcortical regions given its relatively weak signal-to-noise ratio in these areas..$^{12}$ In addition, activity in the thalamus may not be completely removed because the size of the lesion center is too small.

\section{Study Limitations}

The small sample size is the main limitation of this study, although a longitudinal design could have enhanced study sensitivity. Studies with a large number of participants are necessary in the future. Although the current 
study reports longitudinal changes up to 12 months after treatment, further long-term longitudinal studies are necessary to observe the evolution beyond 12 months.

\section{Conclusions}

To the best of our knowledge, this is the first study to investigate the impact of MRgFUS VIM thalamotomy on spontaneous neural activity in medication-refractory tremor-dominant PD. The visual area is, for the first time, reported as relevant to tremor improvement in PD after MRgFUS thalamotomy, suggesting a distant effect of MRgFUS thalamotomy and the involvement of specific visuomotor networks in tremor control in PD. Future studies with larger sample sizes are needed to investigate the potential role of the visual area in predicting tremor improvement after thalamotomy.

\section{Acknowledgments}

This work has been supported by the National Natural Science Foundation of China (81730048, 81825012; X.L.).

\section{References}

1. Armstrong MJ, Okun MS. Diagnosis and treatment of Parkinson disease: a review. JAMA. 2020;323(6):548-560.

2. Marras C, Beck JC, Bower JH, et al. Prevalence of Parkinson's disease across North America. NPJ Parkinsons Dis. 2018;4:21.

3. Hughes AJ, Daniel SE, Blankson S, Lees AJ. A clinicopathologic study of 100 cases of Parkinson's disease. Arch Neurol. 1993;50(2):140-148.

4. Rajput AH, Rozdilsky B, Ang L. Occurrence of resting tremor in Parkinson's disease. Neurology. 1991;41(8):1298-1299.

5. Elias WJ, Shah BB. Tremor. JAMA. 2014;311(9):948-954.

6. Kalia LV, Lang AE. Parkinson's disease. Lancet. 2015; 386(9996):896-912.

7. Hallett M. Parkinson's disease tremor: pathophysiology. Parkinsonism Relat Disord. 2012;18(suppl 1):S85-S86.

8. Xu Y, He Q, Wang M, et al. Safety and efficacy of magnetic resonance imaging-guided focused ultrasound neurosurgery for Parkinson's disease: a systematic review. Neurosurg Rev. 2021;44(1):115-127.

9. Bond AE, Shah BB, Huss DS, et al. Safety and efficacy of focused ultrasound thalamotomy for patients with medication-refractory, tremor-dominant Parkinson disease: a randomized clinical trial. JAMA Neurol. 2017;74(12):14121418.

10. Schlesinger I, Eran A, Sinai A, et al. MRI guided focused ultrasound thalamotomy for moderate-to-severe tremor in Parkinson's disease. Parkinsons Dis. 2015;2015:219149.

11. Zur G, Lesman-Segev OH, Schlesinger I, et al. Tremor relief and structural integrity after MRI-guided focused US thalamotomy in tremor disorders. Radiology. 2020;294(3): 676-685.

12. Zang YF, He Y, Zhu CZ, et al. Altered baseline brain activity in children with ADHD revealed by resting-state functional MRI. Brain Dev. 2007;29(2):83-91.

13. Kiviniemi V, Jauhiainen J, Tervonen O, et al. Slow vasomotor fluctuation in fMRI of anesthetized child brain. Magn Reson Med. 2000;44(3):373-378.

14. Li Z, Zhu Y, Childress AR, et al. Relations between BOLD fMRI-derived resting brain activity and cerebral blood flow. PLoS One. 2012;7(9):e44556.

15. Zou QH, Zhu CZ, Yang Y, et al. An improved approach to detection of amplitude of low-frequency fluctuation (ALFF) for resting-state fMRI: fractional ALFF. J Neurosci Methods. 2008;172(1):137-141.

16. Guo T, Guan X, Zeng Q, et al. Correlations between CSF proteins and spontaneous neuronal activity in Parkinson's disease. Neurosci Lett. 2018;673:61-66.

17. Tang Y, Meng L, Wan CM, et al. Identifying the presence of Parkinson's disease using low-frequency fluctuations in BOLD signals. Neurosci Lett. 2017;645:1-6.

18. Hu XF, Zhang JQ, Jiang XM, et al. Amplitude of low-frequency oscillations in Parkinson's disease: a 2-year longitudinal resting-state functional magnetic resonance imaging study. Chin Med J (Engl). 2015;128(5):593-601.

19. Jankovic J, McDermott M, Carter J, et al. Variable expression of Parkinson's disease: a base-line analysis of the DATATOP cohort. Neurology. 1990;40(10):1529-1534.

20. Wang TR, Bond AE, Dallapiazza RF, et al. Transcranial magnetic resonance imaging-guided focused ultrasound thalamotomy for tremor: technical note. Neurosurg Focus. 2018; 44(2):E3

21. Elias WJ, Lipsman N, Ondo WG, et al. A randomized trial of focused ultrasound thalamotomy for essential tremor. $N \mathrm{Engl}$ J Med. 2016;375(8):730-739.

22. Gallay MN, Moser D, Rossi F, et al. MRgFUS pallidothalamic tractotomy for chronic therapy-resistant Parkinson's disease in 51 consecutive patients: single center experience. Front Surg. 2020;6:76.

23. Zaaroor M, Sinai A, Goldsher D, et al. Magnetic resonanceguided focused ultrasound thalamotomy for tremor: a report of 30 Parkinson's disease and essential tremor cases. J Neurosurg. 2018;128(1):202-210.

24. Stacy MA, Elble RJ, Ondo WG, et al. Assessment of interrater and intrarater reliability of the Fahn-Tolosa-Marin Tremor Rating Scale in essential tremor. Mov Disord. 2007;22(6): 833-838.

25. Song XW, Dong ZY, Long XY, et al. REST: a toolkit for resting-state functional magnetic resonance imaging data processing. PLoS One. 2011;6(9):e25031.

26. Cordes D, Haughton VM, Arfanakis K, et al. Frequencies contributing to functional connectivity in the cerebral cortex in "resting-state" data. AJNR Am J Neuroradiol. 2001;22(7): $1326-1333$.

27. Wintermark M, Druzgal J, Huss DS, et al. Imaging findings in MR imaging-guided focused ultrasound treatment for patients with essential tremor. AJNR Am J Neuroradiol. 2014; 35(5):891-896.

28. Skidmore FM, Yang M, Baxter L, et al. Reliability analysis of the resting state can sensitively and specifically identify the presence of Parkinson disease. Neuroimage. 2013;75:249-261.

29. Hou Y, Wu X, Hallett M, et al. Frequency-dependent neural activity in Parkinson's disease. Hum Brain Mapp. 2014; 35(12):5815-5833.

30. Chen HM, Wang ZJ, Fang JP, et al. Different patterns of spontaneous brain activity between tremor-dominant and postural instability/gait difficulty subtypes of Parkinson's disease: a resting-state fMRI study. CNS Neurosci Ther. 2015; 21(10):855-866.

31. Jang C, Park HJ, Chang WS, et al. Immediate and longitudinal alterations of functional networks after thalamotomy in essential tremor. Front Neurol. 2016;7:184.

32. Wintermark M, Huss DS, Shah BB, et al. Thalamic connectivity in patients with essential tremor treated with MR imaging-guided focused ultrasound: in vivo fiber tracking by using diffusion-tensor MR imaging. Radiology. 2014;272(1): 202-209.

33. Zhang D, Liu X, Chen J, et al. Widespread increase of functional connectivity in Parkinson's disease with tremor: a resting-state FMRI study. Front Aging Neurosci. 2015;7:6.

34. Zhang D, Liu X, Chen J, Liu B. Distinguishing patients with Parkinson's disease subtypes from normal controls based on 
functional network regional efficiencies. PLoS One. 2014; 9(12):e115131.

35. Göttlich M, Münte TF, Heldmann M, et al. Altered resting state brain networks in Parkinson's disease. PLoS One. 2013; 8(10):e77336.

36. Rodriguez-Rojas R, Pineda-Pardo JA, Martinez-Fernandez $\mathrm{R}$, et al. Functional impact of subthalamotomy by magnetic resonance-guided focused ultrasound in Parkinson's disease: a hybrid PET/MR study of resting-state brain metabolism. Eur J Nucl Med Mol Imaging. 2020;47(2):425-436.

37. Hou Y, Ou R, Yang J, et al. Patterns of striatal and cerebellar functional connectivity in early-stage drug-naïve patients with Parkinson's disease subtypes. Neuroradiology. 2018; 60(12):1323-1333.

38. Glickstein M. How are visual areas of the brain connected to motor areas for the sensory guidance of movement? Trends Neurosci. 2000;23(12):613-617.

39. Tuleasca C, Régis J, Najdenovska E, et al. Visually-sensitive networks in essential tremor: evidence from structural and functional imaging. Brain. 2018;141(6):e47.

40. Tuleasca C, Witjas T, Najdenovska E, et al. Assessing the clinical outcome of Vim radiosurgery with voxel-based morphometry: visual areas are linked with tremor arrest! Acta Neurochir (Wien). 2017;159(11):2139-2144.

41. Tuleasca C, Witjas T, Van de Ville D, et al. Right Brodmann area 18 predicts tremor arrest after Vim radiosurgery: a voxel-based morphometry study. Acta Neurochir (Wien). 2018; 160(3):603-609.

42. Tuleasca C, Najdenovska E, Régis J, et al. Clinical response to Vim's thalamic stereotactic radiosurgery for essential tremor is associated with distinctive functional connectivity patterns. Acta Neurochir (Wien). 2018;160(3):611-624.
43. Tuleasca C, Bolton TAW, Régis J, et al. Normalization of aberrant pretherapeutic dynamic functional connectivity of extrastriate visual system in patients who underwent thalamotomy with stereotactic radiosurgery for essential tremor: a resting-state functional MRI study. J Neurosurg. 2019;132(6): 1792-1801.

44. Saint-Cyr JA, Ungerleider LG, Desimone R. Organization of visual cortical inputs to the striatum and subsequent outputs to the pallido-nigral complex in the monkey. J Comp Neurol. 1990;298(2):129-156.

\section{Disclosures}

The authors report no conflict of interest concerning the materials or methods used in this study or the findings specified in this paper.

\section{Author Contributions}

Conception and design: Lou, Xiong. Acquisition of data: Xiong, He, Zong, Bian, Zhang. Analysis and interpretation of data: Xiong, Duan, Zhou. Drafting the article: Xiong. Critically revising the article: Lou, Han, Zhou. Reviewed submitted version of manuscript: Lou. Approved the final version of the manuscript on behalf of all authors: Lou. Statistical analysis: Xiong, Han, Duan, Zhou. Administrative/technical/material support: Lou, Pan. Study supervision: Lou, Pan.

\section{Correspondence}

Xin Lou: Chinese PLA General Hospital, Beijing, China. louxin@301hospital.com.cn. 